\title{
Perforating uterine choriocarcinoma - a rare case report
}

\begin{abstract}
Gestational Trophoblastic disease is a tumour that is more sensitive and respond well to a wide variety of chemotherapeutic regimes. However emergency hysterectomy is still required for life threatening complications associated with GTD.
\end{abstract}

Keywords: Choriocarcinoma, Uterine perforation, Gestational Trophoblastic disease, Bleeding per vaginum

\author{
Volume 7 Issue 4 - 2017 \\ Saxena Pinkee, Zeepee Godha, Laul Poonam, \\ Kadam KVijay \\ Deen Dayal Upadhaya Hospital, India
}

\begin{abstract}
Correspondence: Zeepee Godha, Senior Resident Obs \& Gyn Deen Dayal Upadhaya Hospital, New Delhi, India,Tel 7827485827,Email zeepee_dr@yahoo.com.in
\end{abstract}

Received: November 28, 2016 | Published: August 02, 2017

\section{Case report}

\section{Introduction}

Gestational Trophoblastic disease (GTD) is a group of unique benign and malignant tumours derived from the human placenta. These groups of tumours are more sensitive and usually respond well to a wide variety of chemotherapeutic regimes. In few advanced cases GTDs can lead to surgical emergencies requiring hysterectomy. GTD also have been reported being complicated by the increased occurrence of secondary primary tumors. We are presenting a rare case of perforating uterine choriocarcinoma with life threatening complications.

\section{Case presentation}

A 30 yrs female G8P4L4A3 presented with bleeding per vaginum on and off for the last six months and pain in abdomen for one day. Six months back she had amenorrhea for one and half month with positive urine pregnancy test for which she took pills to medically terminate the pregnancy. After that she had bleeding for 7 days. She had repeat episode of bleeding per vaginum after a period of amenorrhea for 2 months for which dilatation and evacuation was done in a private hospital. Again a repeat dilatation and evacuation was done 25 days later. But her irregular bleeding continued and she reported to us after 15 days after that for further management. On Admission she was pale, pulse - 112/min, BP- 90/60 mm Hg. Per abdomen soft distension was present with guarding and mild tenderness. Per speculum examination revealed minimal bleeding through cervix and on per vaginal examination uterus was retroverted, 8 weeks size with cervical motion tenderness and fullness in pouch of douglas.

Her haemoglobin was $6.3 \mathrm{gm} / \mathrm{dl}$ and the rest of her blood investigations were normal. Urine pregnancy was still positive with $\beta$-HCG-2,11,000 IU $/ \mathrm{ml}$. Chest X-ray was normal. Ultrasound revealed bulky uterus with lesion measuring $25 \times 23 \mathrm{~mm}$ on posterior wall with increased vascularity. Bilateral adnexa was normal with free fluid present in POD. Patient was planned for emergency laparotomy after arranging three unit packed red cells. Injection methotrexate $50 \mathrm{mg}$ IM given stat. Per operative $1200 \mathrm{cc}$ of haemoperitoneum was present, uterus was 8 weeks in size with $3 \mathrm{~cm}$ perforation seen at fundus with polypoidal mass protruding at through it (Figure $1 \& 2$ ).

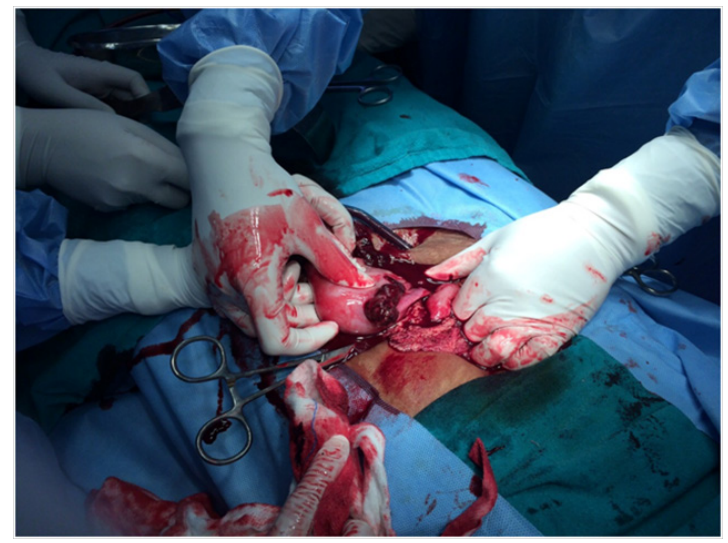

Figure I Hemoperitonium.

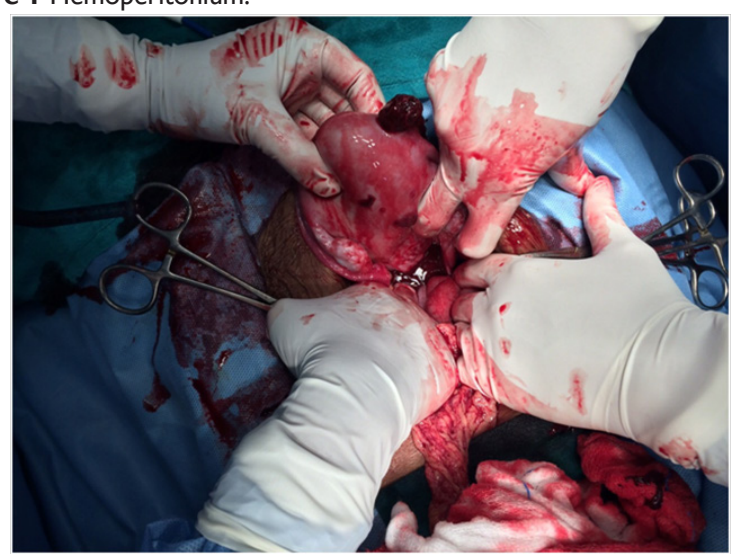

Figure $\mathbf{2}$ Lesion seen perforating at fundus.

Bilateral tubes and ovaries were normal. Rest of the abdomen was normal. Hysterectomy was done due to excessive bleeding and friability of the tissues. Tissue was sent for histopathology. HPE revealed feature suggestive of choriocarcinoma. Postoperative period was uneventful and her $\beta$-HCG was 46,054 IU/ml 48hours after surgery.

She was given single agent chemotherapy with methotrexate. After completion of first cycle of chemotherapy her $\beta$-HCG was 18,299IU/ 
$\mathrm{ml}$. Her $\beta$-HCG after completion of second cycle of chemotherapy$1,01,067 \mathrm{iu} / \mathrm{ml}$. Patient diagnosed to be resistant to single drug regime and hence started on EMACO. Patient has received five doses of chemotherapy after which her $\beta$-HCG is normal. She is currently under follow up.

\section{Discussion}

Gestational Trophoblastic disease (GTD) is a group of unique benign and malignant tumours derived from the human placenta. Choriocarcinoma is a rare malignancy with an incidence of 1 in 40,000 pregnancies. It is characterized by abnormal trophoblastic hyperplasia and anaplasia. There is absence of villi and presence of areas of hemorrhage and necrosis. In $50 \%$ of the cases it is preceded by hydatidiform mole and in the rest by normal pregnancy, abortion and ectopic pregnancy. These groups of tumours are more sensitive and respond well to a wide variety of chemotherapeutic regimes. However role of surgery for such patients is still essential as life saving procedure for life threatening complications like perforation and haemorrhage. In our case the patient presented to us with haemorrhagic shock with the tumor perforating the uterus. The diagnosis of GTD was missed earlier by others who performed repeated dilatation and evacuation for it. This reiterates the need of high index of suspicion to diagnose this condition. ${ }^{1}$ Choriocarcinoma should be suspected when there is abnormal uterine bleeding following an abortion or hydatidifo mole. Hysterectomy may be necessary to control uterine hemorrhage or sepsis, or to resect resistant disease. ${ }^{2,3}$

Single-dose methotrexate regimen have been given in the treatment of low-risk gestational trophoblastic neoplasia with success rate of $77 \% .{ }^{4}$ Our case was one of few which failed to respond to single agent chemotherapy and required multidrug regim.

\section{Conclusion}

In conclusion a high index of suspicion is often necessary to diagnose choriocarcinoma which can present with life threatening complications. Hysterectomy is still required in these chemosensitive tumor.

\section{Acknowledgments}

None.

\section{Conflicts of interest}

None.

\section{References}

1. Dasari P. Missing choriocarcinoma may be easy but not suspecting it in a high-risk case can be potentially fatal. BMJ Case. Rep 2015. pii:bcr2014208526

2. Topuz S, Iyibozkurt $\mathrm{C}$, Mete $\mathrm{O}$, et al. Life-saving hysterectomy in choriocarcinoma: presentation of two cases. Eur J Gynaecol Oncol. 2008;29(6):664-665.

3. Alazzam M, Hancock BW, Tidy J. Role of hysterectomy in managing persistent gestational trophoblastic disease. $J$ Reprod Med. 2008;53(7):519-524.

4. Matsui H, Suzuka K, Yamazawa K, et al. Relapse rate of patients with low-risk gestational trophoblastic tumor initially treated with singleagent chemotherapy. Gynecol Oncol. 2005;96(3):616-620. 